\title{
A Note on Japanese Names and Terms
}

Diacritics signifying long vowels are essential in romanizing Japanese and are indicated here with $\bar{O}, \bar{o}$, and $\bar{u}$, though the diacritics are dropped in well-known place names. The usage followed is usually that in the Kenkyusha New Japanese-English Dictionary. Terms other than proper nouns or words in common use in English are italicized. Names of Japanese individuals are usually given in the Japanese order-family names first. See the Glossary and Biographical Notes for other potential confusions. 
